Conclusions: In Spain FS hospital admissions in patients with RA decreased between 1999-2015 with an estimation of $0,5 \%$ annual reduction not statistically significative.

Disclosure of Interest: None declared

DOI: 10.1136/annrheumdis-2018-eular.4755

\section{FRI0068 ULTRASONOGRAPHIC SUBCLINICAL CARDIOVASCULAR FINDINGS IN HISPANIC RA PATIENTS: A CASE-CONTROL STUDY}

J.R. Azpiri-López ${ }^{1}$, D.A. Galarza-Delgado ${ }^{2}$, I.J. Colunga-Pedraza², A.H. GuillénLozoya ${ }^{2}$, J.A. Dávila-Jiménez ${ }^{2}$, E.E. Abundis-Márquez ${ }^{2} .{ }^{1}$ Cardiology,

${ }^{2}$ Rheumatology, Hospital Universitario "Dr José E. Gonzalez", UANL, Monterrey, Mexico

Background: Patients with RA have a 1.5-2.0 fold increased risk of developing coronary artery disease (CAD) compared with the general population. Patients with RA are typically managed by several physicians, and coordination of care may be suboptimal. ${ }^{1}$ The leading cause of death in RA-patients is atherosclerotic cardiovascular disease (ASCVD). ${ }^{2}$ Carotid artery and heart evaluation by ultrasound is a useful tool for detection of cardiovascular conditions.

Objectives: To compare the presence of subclinical cardiovascular conditions among Mexican-mestizo RA patients and matched controls.

Methods: Design: Observational, cross-sectional, case-control study. Patients of 40 to 75 years old who fulfilled the 2010 ACR/EULAR and/or the 1987 ACR classification criteria for RA were consecutively enrolled. Patients with previous ASCVD or any other rheumatic disease were excluded. Two board-certified radiologist performed and assessed all carotid ultrasounds (cUS) and two board-certified cardiologist performed and assessed transthoracic echocardiography (TTE) according to guidelines of the American Society of Echocardiography. ASE, 2016 Results: The RA patients showed higher prevalence of cardiovascular risk conditions with respect to control population, such as concentric remodelling/hypertrophy, diastolic dysfunction, valve regurgitation and Carotid intima media thickness (CIMT) (See table 1).

Abstract FRI0068 - Table 1. Ecographic cardiovascular patological conditions among RA patients.

\begin{tabular}{|c|c|c|c|c|}
\hline Ecographic findings & $\underset{n=50(\%)}{R A}$ & $\begin{array}{c}\text { Controls } \\
n=50(\%)\end{array}$ & $P$ & O.R. (C.I. 95\%) \\
\hline Valve regurgitation $n \%$ & $42(84)$ & $32(46)$ & 0.023 & $2.95(1.14-7.64)$ \\
\hline Aortic, $n(\%)$ & $4(8)$ & $3(6)$ & 0.999 * & $1.36(0.28-6.42)$ \\
\hline Tricuspid n $n \%$ & $41(82)$ & $29(58)$ & 0.009 & $3.29(1.32-8.23)$ \\
\hline Mild, $n(\%)$ & $37(74)$ & $29(58)$ & 0.091 & $2.06(0.88-4.80)$ \\
\hline Moderate, $n(\%)$ & $4(8)$ & $0(0)$ & $0.126 *$ & $0.92(0.84-0.99)$ \\
\hline Mitral, $n \% \%$ & $28(56)$ & $6(12)$ & 0.0001 & $9.30(3.36-25.87)$ \\
\hline Pulmonar, $n(\%)$ & $8(16)$ & $2(4)$ & 0.046 & $4.57(0.91-22.73)$ \\
\hline Diastolic disfunction n $n \%$ & $34(68)$ & $16(32)$ & 0.003 & $3.46(1.52-7.90)$ \\
\hline LVCR n \% & $13(26)$ & $6(12)$ & 0.074 & $2.50(0.89-7.44)$ \\
\hline LVH, n\% & $5(10)$ & l(2) & 0.092 & $5.40(0.61-48.39)$ \\
\hline LVCRLVH, $n \%$ & $18(36)$ & $8(16)$ & 0.017 & $3.09(1.19-7.99)$ \\
\hline Mass index $(g / m 2)$, mean $\pm S D$ & $66.75 \pm 17.40$ & $73.11 \pm 22.49$ & 0.132 & $1.01(-14.67-1.93)$ \\
\hline LVEF $\%$, mean $=S D$ & $60.14 \pm 5.12$ & $63.42 \pm 5.67$ & 0.004 & $1.12(-5.49-1.06)$ \\
\hline$G L S(\%)$ mean $\pm S D$ & $-20.73 \pm 2.90$ & $-21.03 \pm 2.48$ & 0.639 & $0.96(-0.96-1.56)$ \\
\hline TAPSE $(m m)$, mean $\pm S D$ & $23.01 \pm 4.41$ & $\begin{array}{l}24.06 \pm 3.12 \\
24.12\end{array}$ & 0.193 & $1.05(-2.6-0.54)$ \\
\hline CAWH $n \%$ \% & $21(42)$ & $10(20)$ & 0.017 & $2.80(1.18-7.06)$ \\
\hline Carotid plaque, $n \%$ & II (22) & $10(20)$ & 0.806 & $1.12(0.43-2.95)$ \\
\hline
\end{tabular}

$\mathrm{P}$ (P value) O.R. (Odds ratio) C.I. (Confidence interval) LVCR (Left ventricle concentric remodelation) LVH (Left Ventricle hypertrophy) LVEF (Left ventricle ejection fraction GLS (Global longitudinal strain) TAPSE (Tricuspid annular plane systolic excursion) CAWH (Carotid artery wall hypertrophy) SD (Standard deviation) * (Yates continuity correction)

Conclusions: This study reinforces the usefulness of cUS and TTE as a tool in the evaluation of cardiovascular conditions in RA patients. A systematic search of extra-articular and cardiovascular comorbidities should be mandatory in all RA patients.

\section{REFERENCES:}

[1] Crowson CS, Liao KP, Davis JM, Solomon DH, Matteson EL, Knutson KL, Gabriel SE. Rheumatoid arthritis and cardiovascular disease. American heart journal 2013;166(4):622-628

[2] Avina-Zubieta JA, Thomas J, Sadatsafavi M, Lehman AJ, Lacaille D. Risk of incident cardiovascular events in patients with rheumatoid arthritis: a meta-analysis of observational studies. Annals of the rheumatic diseases 2012;71(9):1524-9.

Acknowledgements: None

Disclosure of Interest: None declared

DOI: 10.1136/annrheumdis-2018-eular.7112

\section{FRI0069 \\ DIFFERENCE OF CLINICAL IMPLICATION REGARDING THE NUMBERS OF AUTOANTIBODIES AT PRESENTATION IN KOREAN PATIENTS WITH EARLY RHEUMATOID ARTHRITIS}

J.Y.Pyo ${ }^{1}$, C.-H. Mun ${ }^{2}$, S.M. Jung ${ }^{2}$, S.W. Lee ${ }^{2}$, J. Song ${ }^{2}$, S.K. Lee ${ }^{2}$, Y.B. Park ${ }^{2}$ ${ }^{1}$ Department of Internal Medicine, Yonsei University Wonju College of Medicine, Wonju; ${ }^{2}$ Department of Internal Medicine, Yonsei University College of Medicine Seoul, Korea, Republic Of

Background: Epitope spreading occurs prior to the clinical diagnosis of rheumatoid arthritis (RA), the number of peptide recognised and its titer increases remarkably as becoming close to the clinical diagnosis. However, the effect of multiple autoantibodies on the clinical outcome is not well known.

Objectives: This study is to investigate the association between the number of autoantibodies at presentation and the clinical aspects in Korean patients with early RA.

Methods: The number of baseline autoantibody (rheumatoid factor, anticitrullinated peptide antibodies (ACPA), and anti-carbamylated protein antibodies (antiCarP)) was analysed in the Korean Intensive Management of Early Rheumatoid Arthritis (KIMERA) cohort. All patients were disease-modifying antirheumatic drug (DMARD)-naïve RA patients with symptom duration less than 1 year. They were intensively treated by adjusting medications every 4 weeks, and treated to target as DAS28 of $<2.6$. Patients were classified regarding the number of autoantibod ies and clinical characteristics were compared between the groups.

Results: A total of 128 patients were included, seronegative patients showed higher baseline physician global VAS ( 7.3 vs 4.7 vs 4.6 vs $4.4, p=0.005)$ and DAS28-ESR (7.1 vs 4.5 vs 5.0 vs $4.7, p=0.003$ ) than patients with 1,2 , and 3 autoantibodies. Also seronegative patients showed a trend to have higher baseline $\mathrm{HAQ}$ score and pain VAS. After intensive treatment, there were no differences in remission rate and DAS28-ESR at 12,24 , and 36 months.

Abstract FRI0069 - Table 1. Disease characteristics regarding number of autoantibodies

\begin{tabular}{lccccc}
\hline Variables & $\mathbf{0}(\mathbf{n}=\mathbf{5})$ & $\mathbf{1}(\mathbf{n}=\mathbf{1 3})$ & $\mathbf{2}(\mathbf{n}=\mathbf{5 8})$ & $\mathbf{3}(\mathbf{n}=\mathbf{5 2})$ & $\mathbf{p}$-value \\
\hline Age, years & $58.2 \pm 16.2$ & $46.4 \pm 12.4$ & $52.8 \pm 12.9$ & $48.2 \pm 13.5$ & 0.104 \\
Female sex, N (\%) & $5(100 \%)$ & $7(53.8 \%)$ & $44(75.9 \%)$ & $44(84.6 \%)$ & 0.062 \\
Symptom duration, days & $7.0 \pm 4.8$ & $10.2 \pm 12.1$ & $10.5 \pm 16.0$ & $8.1 \pm 7.5$ & 0.736 \\
BMI (kg/m $\left.{ }^{2}\right)$ & $24.1 \pm 4.2$ & $21.7 \pm 1.9$ & $23.0 \pm 2.9$ & $23.0 \pm 3.1$ & 0.508 \\
ESR (mm/hour) & $59.0 \pm 15.8$ & $44.4 \pm 17.6$ & $56.8 \pm 32.0$ & $57.9 \pm 32.2$ & 0.543 \\
CRP (mg/L) & $20.9 \pm 17.3$ & $13.6 \pm 26.9$ & $19.0 \pm 29.4$ & $12.9 \pm 15.7$ & 0.559 \\
HAQ score & $1.4 \pm 0.5$ & $0.6 \pm 0.6$ & $0.7 \pm 0.6$ & $0.7 \pm 0.6$ & 0.092 \\
Pain VAS & $7.6 \pm 0.9$ & $4.0 \pm 2.0$ & $4.5 \pm 2.7$ & $4.4 \pm 2.9$ & 0.073 \\
Patient global VAS & $7.3 \pm 1.7$ & $4.7 \pm 2.2$ & $4.6 \pm 2.5$ & $4.4 \pm 2.7$ & 0.127 \\
Physician global VAS & $7.0 \pm 0.9$ & $4.9 \pm 1.1$ & $5.5 \pm 1.2$ & $4.5 \pm 1.6$ & 0.005 \\
DAS28-ESR & $7.1 \pm 0.6$ & $4.5 \pm 1.2$ & $5.0 \pm 1.4$ & $4.7 \pm 1.4$ & 0.003 \\
\hline
\end{tabular}

Values are expressed as means \pm standard deviation or $\mathrm{N}(\%)$. BMI, body mass index; ESR, erythrocyte sedimentation rate; $C R P, C$-reactive protein; $H A Q$ : health assessment questionnaire; VAS, visual analogue scale; DAS, disease activity score

\section{Changes in DAS28 according to number of autoantibodies}

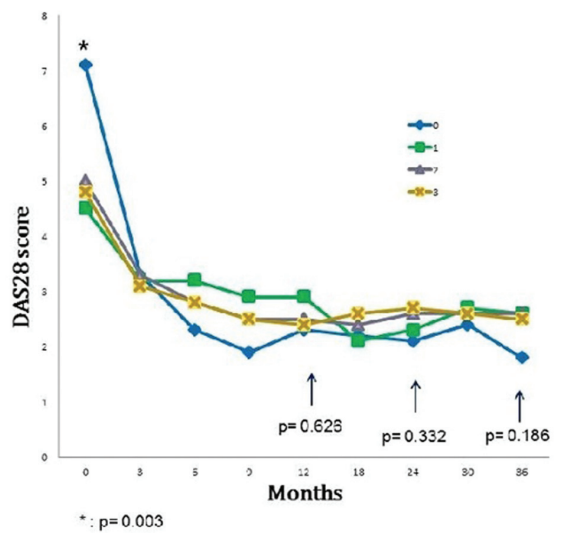

Conclusions: Seronegative patients showed higher levels of baseline inflammation than seropositive patients. However, they had favourable treatment response with rapid improvement which resulted in excellent clinical outcome.

Disclosure of Interest: None declared

DOI: 10.1136/annrheumdis-2018-eular.4354 